\title{
A proactive health plan: taking action on tobacco control
}

\author{
George J Isham
}

It is no secret that the health of most Americans is not as good as it could be. Quality of care is poor, health equity is low, and our health system is in crisis. For health organisations, this myriad of challenges and the massive scope of the problem makes it difficult to prioritise health improvement initiatives. At HealthPartners, a Minnesota based health care organisation with 800000 members, we have defined our initiatives by looking at the health of our members and our community and what we want the future to reflect in terms of good health and organisational values. At the heart of one of our key initiatives is tobacco control.

\section{Goal setting}

Our mission at HealthPartners is to improve the health of our members and the community. To achieve this, we must be competent not only in managing disease, but also in managing the major risk factors for disease. In 1993, we began thinking about how to best identify and affect these risk factors.

Our first step was to survey our population for risk factors for illness such as tobacco use, dietary indiscretion, and inactivity. This drew attention to the fact that these risk factors were a problem for HealthPartners' enrolled population. Next we established a model that illustrated the linkage of risk factors to disease and emphasised the opportunities for intervention. For example, for a person with high blood pressure, a low sodium diet and increased exercise could be an early intervention point. If high blood pressure is not dealt with effectively, heart disease may result, and so on. The objective of this model was to show that we must have interventions and programs along the whole spectrum from being well, to being well with risk factors, to having established disease.

Our third step was to set eight compelling goals for HealthPartners. The first was to reduce the number of cardiac events in our system by $25 \%$. We also set goals for diabetes, immunisation, dental health, breast cancer, prenatal outcomes, childhood injury, and violence. Our hope in creating these goals was to call on individuals across HealthPartners and the region we serve to think about and take action on these issues. Our goal setting quickly led us to tobacco control as we looked for ways to reduce cardiac events.

HealthPartners, 8100 34th Avenue South, PO Box 1309, Minneapolis, MN 55440-1309, USA; George.J.Isham@ HealthPartners.com G Isham
Our fourth step was to engage our members. With an enrolled population, we have the opportunity to evaluate our members through a system wide health risk assessment. We had to devise a method for evaluating their health status and their risks. To do this, we used the Prochaska readiness to change model $^{1}$ for characterising their state of willingness to change when they had risk factors.

We asked our members if they would participate in a health risk assessment and if they would allow their name and smoking status to be passed on to their doctors; $85 \%$ said yes. Furthermore, $65 \%$ said they would actually spend some time helping us design the program. This indicated a high degree of interest.

Drawing on this information, we set up an automated telephone response system for the health risk assessment and linked it to the enrollment of specific employer groups and workplace interventions for employers who were interested. For those employers that wanted to work with us on this, we sent each of their employees an invitation and incentive to participate. We are now working with over 200 employer groups on various phases of this program.

\section{Designing a data system}

Our next step was to design a data system to handle this information. First, we took the information from HealthPartners' databases, such as claims, lab data, pharmacy data, membership and enrollment, and encounter data. We then drew out data that related to the issues we were focusing on (for example, people with diabetes) and then looked at attributes associated with these populations (for example, people with diabetes who smoke). We then fed the health risk assessment data into this, and added other data sources such as chart reviews, pilot studies, and surveys. Next, we took this information to the clinic level. To assist providers in front line clinics, we provided telephone support and educational programs. We have also tried to give our physicians only what they really needed to act on so as not to overwhelm them with too much information.

\section{Guidelines}

We developed guidelines that apply to a number of our goals through the Institute for Clinical Systems Integration (ICSI), a collaborative of about 20 medical groups serving HealthPartners' members. ICSI develops and implements guidelines and is dedicated to improving quality of care.

Our guidelines for heart disease, for example, include diagnosis of chest pain, the treatment of myocardial infarction, the 
treatment of stable coronary artery disease, and tobacco prevention and cessation. The Agency for Health Care Policy and Research guideline on tobacco ${ }^{2}$ has been important in the development of the ICSI guidelines on tobacco.

\section{Measurement}

We have used several approaches for evaluation. We have used the Health Plan Employer Data and Information Set (HEDIS) indicators; however, they were not designed for internal measurement in medical groups. We have also looked at what we call clinical indicators that we have developed for our medical groups. In some cases these are similar to HEDIS indicators, while in others they are different. Tobacco ask-and-advise rates have been useful in monitoring the awareness of tobacco use in patients in the clinic and the rates at which cessation advice has been given. Monitoring prevalence rates in the population allow us to evaluate overall progress. To communicate results, we displayed some measures of performance on our web site at http://www.healthpartners.com

\section{Incentives}

We created a recognition program to reward progress made by the medical groups. This unique strategy financially recognised the performance of our medical groups in achieving our objectives in terms of behaviour change and outcomes. Specific bonus payments are made to the medical groups for the achievement of performance targets. One key to the success of this program was that we kept the measures and recognition simple.

\section{Advertising to teenagers}

Minnesota has a high and increasing teen smoking rate. To help counter this unfortunate trend, we launched an advertising campaign targeted to teens. In 1995, HealthPartners conducted shopping mall interviews with teens to determine what kinds of messages would dissuade them from starting smoking. We used what we learned from the teens to launch the "garbage face" campaign, a series of advertisements designed to sensitise kids to the ugly effects of smoking. The key messages were that smoking gives teens bad breath and makes them unpopular with the opposite sex. The campaign, which won the American Associa- tion of Health Plan's Community Health Promotion Award, serves as a model for managed care to intervene in the prevention arena and at the community level.

\section{Adult programming}

For its adult population, HealthPartners has made tobacco use a "vital sign" in its clinics and introduced financial incentives to encourage clinical staff to advise smokers to quit. HealthPartners, in partnership with Blue Cross/Blue Shield of Minnesota, is one of the managed care organisations to be awarded a full evaluation grant under the "Addressing Tobacco in Managed Care" research initiative. The grant allows HealthPartners to evaluate a new smoking cessation benefit covering pharmacological aids, combined with an innovative, consumer oriented campaign to raise consumer awareness of this benefit and interest in smoking cessation.

\section{A vision}

To move forward in improving national health, we must continue thinking about what the critical issues are for health systems. As important as it is to engage front line practitioners in terms of making this happen one patient at a time, it is also important to think about how we engage organisations in providing an environment to enable that. At HealthPartners, we have found we need to segment the population in order to target our resources. We have identified the individuals to target, and then successfully intervened with the appropriate resources. It is really a very simple program and one that could be applied to nearly any health system interested in tobacco control or other health improvement initiatives.

George Isham is medical director and chief health officer of HealthPartners. In addition, he is co-chair of the NCQA's committee on performance measurement. This is a committee which approved the addition of a tobacco performance measure to the HEDIS 3.0 quality report card. He also serves as a memto the HEDIS 3.0 quality report card. He also serves as a mem-
ber of the AAHP's quality health care committee, and as a ber of the AAHP's quality health care committee, and as a
member of the CDC's "task force" on community preventive member of the CDC's "task force" on community preventive
health services. Dr Isham is chair of the national advisory committee for addressing tobacco in managed care.

1 Prochaska JO, DiClemente CC, Norcross JC. In search of how people change. Applications to addictive behaviors. Am Psychol 1992;47:1102-14.

2 Fiore MC, Bailey WC, Cohen SJ, et al. Smoking cessation. Clinical Practice Guideline No 18. Rockville, Maryland: US Department of Health and Human Services, Public Health Service, Agency for Health Care Policy and Research April 1996. (AHCPR Publication No 960692.); <http://www.text.nlm.nih.gov/ftrs/tocview>. 